\title{
Improving the Performance of Spatial Domain Image Watermarking with High Boost Filter
}

\author{
Mehdi Alirezanejad ${ }^{1 *}$, Saeed Amirgholipour ${ }^{2}$, Vahid Safari ${ }^{3}$, Shahin Aslani ${ }^{4}$ and Moein Arab ${ }^{4}$ \\ 'Department of Computer, Firuzkooh Branch, Islamic Azad University, Firuzkooh, Iran; m_arn2004@yahoo.com \\ 2Department of Computer, Ramsar Branch, Islamic Azad University, Ramsar, I. R. Iran; s.kasmani@iauramsar.ac.ir \\ ${ }^{3}$ Department of Computer, Collage of Engineering, Shiraz branch, Islamic Azad University, Shiraz, I. R. Iran; \\ Saffari@iaushiraz.ac.ir \\ ${ }^{4}$ Department of Computer, Sharif University of technology international campuse, Kish, I. R. Iran; \\ sh_aslani@kish.sharif.edu
}

\begin{abstract}
Authenticating in transaction of digital media becomes an active research field now a day. That is emerging due to the illegal use of digital media and hacking by unauthorized users. Spatial domain based watermarking methods are one of the authentication methods. These methods are not generally robust against intentional or unintentional changes in the images. In this paper, a new method is proposed to recover watermark more precisely in the spatial domain watermarking method. A high-boost filtering is utilized before executing watermark extraction procedure. The watermark information is magnified by preforming this filter. Effectiveness of this filter is proved by several experiment. Experimental results show that extraction performance of correlation based watermarking algorithm is improved by preforming this filter before recovery procedure.
\end{abstract}

Keywords: Digital Image Watermarking, Spatial Domain Watermarking, Correlation Base Watermarking, Sharpening Filter, High Boost Filtering

\section{Introduction}

Nowadays, it is easy to create a copy and distribute the digital media. Therefore, there is a need for secure copyright protection methods for digital media. The watermarking is a valid solution for this issue. In watermarking, some extra information is embedded in digital media invisibly to protect it $^{1}$.

In the design of the watermarking algorithm always, there is a conflict between robustness and fidelity ${ }^{2,3}$. The fidelity is the amount of reduction in signal quality; and the robustness is the ability of the watermark to remain readable after innocent or malicious signal processing operations on the watermarked image.

Generally, there are two main domain for embedding watermark information: the spatial domain or the transform domain. The watermark is embedded directly in pixels in spatial domain watermarking and they are not robust to image compression ${ }^{4-6}$. Transform domain watermarking schemes use the characteristics of transform domain to embed watermark in more robust and imperceptible area. Discrete Cosine Transform $(\mathrm{DCT})^{7-9}$ and Discrete Wavelet Transform (DWT) ${ }^{10-12}$ are two main frequency domains watermarking methods.

In order to augment the strength of Watermarking algorithms, there are two main methods: embedding watermark in more robust places, increase power of watermark retrieval algorithms.

There are two standard methods for augmenting the strength of watermarking algorithms: embedding watermark in robust area and augment the power of extraction algorithms.

Human visual system (HVS) characteristics is used to select most appropriate places in term of resistance

*Author for correspondence 
and transparency. A perceptually optimal quantization matrix for JPEG standard is proposed by Watson ${ }^{13}$. This matrix describes the artifact visibility as a function of DCT frequency, color channel, and display resolution and brightness ${ }^{13}$. A JND profile is defined for an optimal image subband $\operatorname{coder}^{14}$. This profile code determined which signal is imperceptible and which signal is not imperceptible. Perceptibility thresholds of the quantization noise are defined for the linear phase $9 / 7$ wavelet filters ${ }^{15}$. A visual model is proposed to determine the image dependent upper bounds on watermark insertion. These models have been used in a successful manner to achieve imperceptible watermark embedding ${ }^{16}$. JND in the wavelet domain is operated to achieve transparent watermarks of maximum strength ${ }^{17}$. The features of the human visual system (HVS), as well as the masking effect is utilized to estimate the proper watermark signal power for carrying out the watermark embedding through wavelet coefficient modulation ${ }^{18}$. To increase the performance of the spreadspectrum watermarking, a perceptual model is proposed that takes into account the contrast sensitivity and texture masking ${ }^{19}$.

However, in the second method, it is trying to restore the power of the watermarking method with improving the performance of watermark recovery algorithms by preprocessing. The goal of these methods is to boost watermark detection rate by an efficient and simple model based on image enhancement, edge detection and noise magnifying methods.

An improved detector is proposed for detection based on thresholds extracted by statistic rules on which the method relies ${ }^{20}$. Applying blurring filters to a watermarked image before executing watermark detection can increase the possibility of detection ${ }^{21}$. Since blurring filters, suppress the high spatial frequencies, they generally distort the image quality. However, for a watermark that has dominant low frequency content, the application of a blurring filter can serve to improve the statistical environment for watermark detection and thereby improves the detection probability. Since the content of image might interfere with the watermark, especially in the low-frequency parts, the reliability of the detector could be improved by applying matched filtering before correlation ${ }^{6}$. This decreases the influence of the original image to the correlation. Therefore, the watermark could effortlessly be extracted from watermarked image.

As a category of blind embedding watermark, $\mathrm{Hafiz}^{22}$ proposed an approach to blind watermark detection/decoding for spread spectrum by using of Independent Component Analysis theory. It uses the theory of Independent Component Analysis (ICA) and detects the watermark with a blind source separation method. The watermark information is considered as noises. This noise is increased, and then the watermark information is extracted by altering the extracted data from the frequency domain according to the global minimum method ${ }^{23}$. A preprocessing method is proposed that exploit a combination of noise boosting and filtering to facilitate recovering watermark information from watermarked image in the DCT-based watermarking algorithm ${ }^{4,5}$. Recently, the effect of highboost and Laplacian filter is proved by Amirgholipour and Alirezanejad ${ }^{24,25}$. They proposed a new application of sharpening filter for augmenting recovery algorithms in the spatial domain watermarking.

In this paper, a new Application of high boost filtering is presented. In this approach, before watermark extraction, a special type of high boost filter is applied on watermark image, which could be attacked or not, in the spatial domain. It increases the distinction between the watermark and the host image. Thus, the watermark could easily be extracted from the modified watermarked image. Experimental results show that watermark detection reliability is increased by the proposed method in the spatial domain based watermarking.

\section{Proposed Methods}

In this paper, high boost filters is used to increase power of watermark extraction algorithm in watermarking schemes. In this section, at first the filtering and their application are introduced in brief. Then, a definition of high boost filter and its' application according to Gonzalez image processing book ${ }^{26}$ is described. Finally, a reason and motivation of performing this filters is explained.

\subsection{High Boost Filtering}

High boost filter is a filter for sharpening the edge of an image in image processing. High frequency components of image which is representing the images details, could be magnified by this filter. This filter is made from a combination of an all pass filter and an edge detection filter (Laplacian filter). Thus, it highlights edges and results in image sharpener.

A process used for many years in the publishing industry to sharpen images consists of subtracting a 
blurred version of an image from the image itself. This process, called unsharp masking, is expressed as Sample correlation method.

$$
g_{s}(x, y)=g(x, y)-\bar{g}(x, y)
$$

where $\mathrm{g}_{\mathrm{s}}(x, y)$ denotes the sharpened image obtained by unsharp masking, and $\bar{g}(x, y)$ is a blurred version of $\mathrm{g}(x, y)$. The origin of unsharp masking is in darkroom photography, where it consists of clamping together a blurred negative to a corresponding positive film and then developing this combination to produce a sharper image.

A slight further generalization of unsharp masking is called high-boost filtering. A high-boost filtered image, $\mathrm{g}_{\mathrm{hb}}$ is defined at any point $(\mathrm{x}, \mathrm{y})$ as

$$
g_{h b}(x, y)=A g(x, y)-\bar{g}(x, y)
$$

Where $A>=1$ and $\bar{g}$ is a blurred version of $g$. This equation may be written as

$$
g_{h b}(x, y)=(A-1) g(x, y)+g(x, y)-\bar{g}(x, y)
$$

By using equation (3), we obtain a High Boost filtered image.

$$
g_{h b}(x, y)=(A-1) g(x, y)+g_{s}(x, y)
$$

Another way to implement High Boost filtering is using the two masks that shown in equations (5), (6). As the value of $A$ increases past 1, the influence of the sharpening process becomes less and less important. Finally, if A is large enough, the high-boost image will be equal to the original image multiplied by a constant.

$$
\begin{aligned}
& {\left[\begin{array}{ccc}
0 & -1 & 0 \\
-1 & A+4 & -1 \\
0 & -1 & 0
\end{array}\right]} \\
& {\left[\begin{array}{ccc}
-1 & -1 & -1 \\
-1 & A+8 & -1 \\
-1 & -1 & -1
\end{array}\right]}
\end{aligned}
$$

In this paper, a special type of high boost filter is used. It is shown in the $3 \times 3$ convolution matrix looks as follows:

$$
\text { (20) }\left[\begin{array}{lll}
-1 & -1 & -1 \\
-1 & 10 & -1 \\
-1 & -1 & -1
\end{array}\right]
$$

\subsection{Motivation of Utilization of High Boost Filtering}

The goal of this paper is to show the effectiveness of preforming typical high boost filtering on performance of spatial domain watermarking method. This filter is applied on attacked watermarked image before execution of the watermark extraction algorithm. A compression between the application of this filter in this way and the approach presented by deprovere is done. Depovere's method ${ }^{6}$ apply this filter on each block of watermarked image before computing the correlation between it and pseudo random noise corresponding to the watermark bits.

A special kind of edge enhancement operations is preformed that separate watermarked and unwatermarked parts of image. Therefore, performing high boost filtering operation on the watermarked image; which may be attacked by blurring, noise addition, compression, scaling, in the spatial domain watermarking method, makes details of watermarked image (watermark information) to become manifest. It means that watermark information, which is different from the image background, becomes recognizable straightforwardly to the procedure of watermark extraction. Thus, the presented method is better than the filtering technique that its goal is facilitating watermark insertion, because it is simple, blind, and could be applied in the real time.

\subsection{Typical Spatial Domain Watermarking}

The watermark process is shown in Figure 1.Figure 1 show the watermark embedding and extraction process in the spatial domain. These algorithms is explained with details in $^{24,25}$. Correlation based algorithm is used for extraction method. The proposed filtering process is applied before executing the extraction algorithms.

\section{Experimental Results}

The efficiency of the proposed methods on correlation based methods is shown by testing it on two standard grayscale images with $512 \times 512$, these images is shown in Figure 2(a)-(b). Pepper is used as a representation of image with low spatial frequency and Barbara as a representation of image with average spatial frequency. In this experiment, a $32 \times 32$ binary image, as shown in Figure 2 (c) is used as the watermark. Several attacks are done to evaluate the robustness of the proposed watermarking algorithm. 

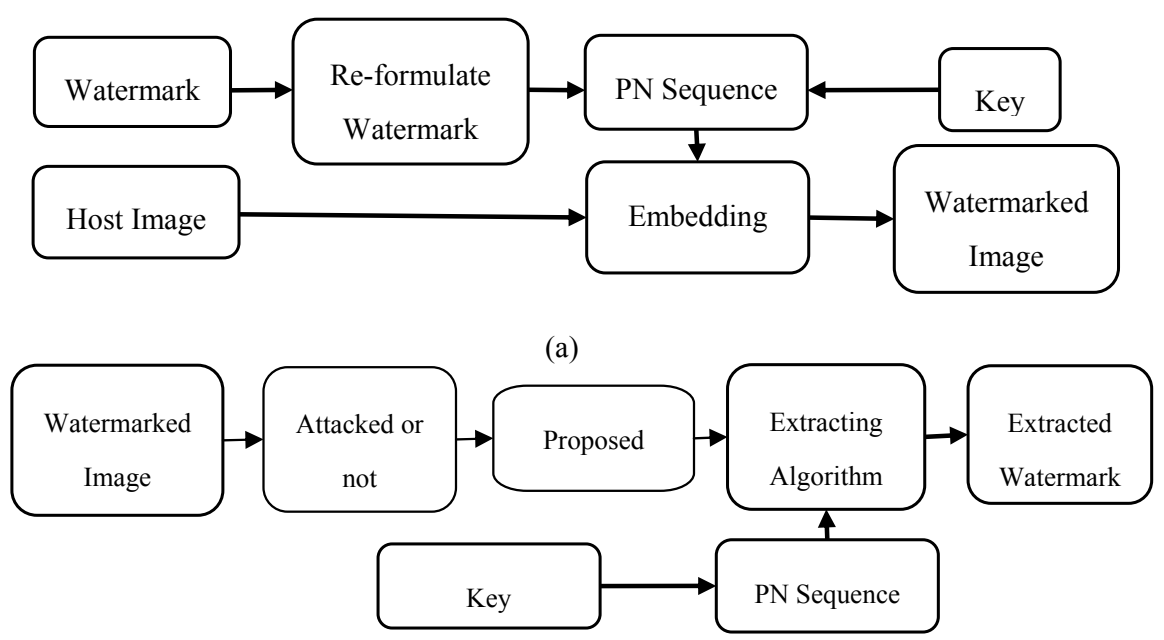

(b)

Figure. 1 (a) The watermark embedding process; (b) The watermark extracting process.

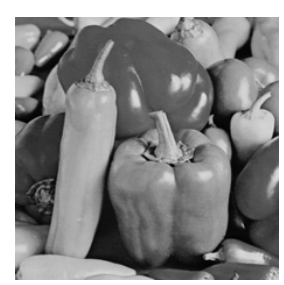

(a)

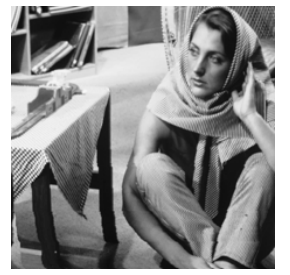

(b)

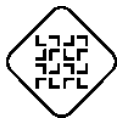

(c)
Figure 2. (a) Standard Peppers image; (b) Standard Barbara image; (c) Watermark.

Peak Signal-to-Noise Ratio (PSNR), is employed to assess the difference between an original image and a watermarked image as an imperceptible measure. The Mean Absolute Error (MAE) measures the difference between an original watermark $\mathrm{W}$ and the corresponding extracted one as robustness capability. If a method has lower MAE, it is more robust. The PSNR and the MAE are, respectively, defined by equations 8 and 9; respectively.

$$
\operatorname{PSNR}(\mathrm{I}, \overline{\mathrm{I}})=10 \log _{10} \frac{255 \times 255}{\frac{\left.\sum_{\mathrm{i}=0 \mathrm{j}=0}^{\mathrm{M}-1 N-1} \sum_{\mathrm{ij}}-\bar{I}_{\mathrm{ij}} \|\right)^{2}}{M \times N}}
$$

And

$$
\operatorname{MAE}(W, \breve{W})=\frac{\sum_{i=0}^{S-1}\left\|w_{i}-\breve{w}_{i}\right\|_{1}}{|W|}
$$

A comparison is made between the proposed method and Depovere's method ${ }^{6}$

To examine the robustness, several attacks are implemented on the watermarked image, including JPEG compression, Gaussian filtering, adding Salt and pepper noise, image scaling. The presented method and experiments are implemented using MATLAB. These experiments results in term of MAE is shown in the Table 1 and Table 2.

The quantitative results in terms of the MAE is provided in the Table 1 and Table 2 . These show the results of implementing the proposed algorithm on the images with PSNR 40 and 35, respectively.

Tables 1 show the result for the watermarked image with PSNR 40. As shown in Tables 1, the proposed performs consistently the best in all experiments, especially for the scailing, jpeg compression, and Gaussian lowpass filtering attacks. In no attack case, the MAE of Barbara and Peppers are .1133, .0850, respectively; and using previous method improve to .1162 , .0840, respectively. In 70\% scaling, the MAE of Barbara and Peppers are $.1035, .1386$, respectively; and using previous method, the improvements are $.0752, .1142$, respectively. When $15 \%$ salt and pepper noise is added to watermarked image, the performance of proposed is very close to former method. In this attack, the MAE of Barbara and Peppers are .1484, .2002, respectively; and using previous method, the improvements are .1396, .2041, respectively. As shown in this table is significantly better than Depovere method. 
Table 1. Performance analysis of correlation based spatial domain watermarking techniques against Attacks (PSNR 40)

\begin{tabular}{|c|c|c|c|c|}
\hline \multirow[b]{2}{*}{ Attack } & \multirow[b]{2}{*}{ Image } & \multicolumn{3}{|c|}{ MAE } \\
\hline & & $\begin{array}{l}\text { Without } \\
\text { Filtering }\end{array}$ & Depvere's & $\begin{array}{c}\text { Proposed } \\
\text { Method }\end{array}$ \\
\hline \multirow{2}{*}{ No Attack } & Barbara & 0.1797 & 0.0635 & 0.0664 \\
\hline & Peppers & 0.0889 & 0.0049 & 0.0039 \\
\hline \multirow{2}{*}{ Jpeg Compression (50\%) } & Barbara & 0.3730 & 0.3135 & 0.3018 \\
\hline & Peppers & 0.3291 & 0.2305 & 0.2100 \\
\hline \multirow{2}{*}{ Scaling (To 70\% Actual Size) } & Barbara & 0.3652 & 0.2900 & 0.2617 \\
\hline & Peppers & 0.2949 & 0.1807 & 0.1563 \\
\hline \multirow{2}{*}{ Noise Addition (15\% salt and pepper noise) } & Barbara & 0.3623 & 0.2227 & 0.2139 \\
\hline & Peppers & 0.3359 & 0.1318 & 0.1357 \\
\hline \multirow{2}{*}{ Gaussian Lowpass Filtering $($ hsize $=5$, Sigma $=1$ ) } & Barbara & 0.3594 & 0.2910 & 0.2764 \\
\hline & Peppers & 0.3252 & 0.2080 & 0.1973 \\
\hline
\end{tabular}

Table 2. Performance analysis of correlation based spatial domain watermarking techniques against Attacks (PSNR 35)

\begin{tabular}{|c|c|c|c|c|}
\hline \multirow[b]{2}{*}{ Attack } & \multirow[b]{2}{*}{ Image } & \multicolumn{3}{|c|}{ MAE } \\
\hline & & $\begin{array}{l}\text { Without } \\
\text { Filtering }\end{array}$ & Depovere's & $\begin{array}{c}\text { Proposed } \\
\text { Method }\end{array}$ \\
\hline \multirow{2}{*}{ No Attack } & Barbara & 0.0879 & 0.0254 & 0.0234 \\
\hline & Peppers & 0.0381 & $\mathbf{0}$ & $\mathbf{0}$ \\
\hline \multirow{2}{*}{ Jpeg Compression (50\%) } & Barbara & 0.2734 & 0.1855 & 0.1729 \\
\hline & Peppers & 0.2051 & 0.0947 & 0.0801 \\
\hline \multirow{2}{*}{ Scaling (To 70\% Actual Size) } & Barbara & 0.3027 & 0.2236 & 0.1982 \\
\hline & Peppers & 0.2285 & 0.1143 & 0.0869 \\
\hline \multirow{2}{*}{ Noise Addition (15\% salt and pepper noise) } & Barbara & 0.2666 & 0.1123 & 0.1143 \\
\hline & Peppers & 0.2363 & 0.0488 & 0.0439 \\
\hline \multirow{2}{*}{ Gaussian Lowpass Filtering $($ hsize $=5$, Sigma $=1)$} & Barbara & 0.3125 & 0.2129 & 0.1992 \\
\hline & Peppers & 0.2637 & 0.1260 & 0.1152 \\
\hline
\end{tabular}

Tables 2 show the result for the watermarked image with PSNR 35. As shown in Tables 2, the proposed performs consistently the best in all experiments, especially for the scailing, jpeg compression, and Gaussian lowpass filtering attacks. . In no attack case, the MAE of Barbara and Peppers are .0645, .0381, respectively; and using previous method, the improvements are .0625 , .0381 , respectively. For example, in $70 \%$ scaling, the MAE of Barbara and Peppers are .1045, .1416, respectively; and using previous method, the improvements are .0791,.1142, respectively. When $15 \%$ salt and pepper noise is added to watermarked image, the performance of proposed is very close to former method. In this attack, the MAE of Barbara and Peppers are .1523, .1924, respectively; and using previous method, the improvements are .1543, .1875 , respectively. As shown in these tables, the results are significantly increase the power of watermark extraction algorithm. As a result, we could use spatial domain watermarking as well as frequency-domain methods in the real applications.

Table 1 and table 2 clearly shows that the proposed method could improve the watermark recovery algorithm. Even it performed better than the former method was presented by Depovere's. 


\subsection{Recommendations for Future Research}

- Future studies are needed to find better filtering method for more augment the performance of watermarking in the spatial domain.

- In the future, the application of this filter in the frequency domain could be investigated in further research.

- Also, it is necessary to compare the spatial domain algorithm with the proposed watermark recovery modification with frequency-domain image watermarking.

- Another important research area is utilizing the combination of different filters for improving the watermark recovery algorithm.

- Application of this filter in digital video watermarking is another challenge. If we could use the ability of this filter in the video watermarking domain for improvement of existing methods, a major breakthrough could be achieved.

\section{Conclusion}

In this paper, a new application of high boost filtering method is presented to obtain more imperceptibility and robustness spatial domain based watermarking schemes. The distinction between watermarked part and unwatermarked part of host image is increased by this filter. Then, this enhanced watermarked image is used as a new one, which is ready for watermark extraction's process. Therefore, proposed method helps details of watermarked image become manifest. Different experiments have shown that presented filtering method could significantly increase power of spatial domain watermark extracting. Effectiveness of the proposed filtering method is verified by comparing its result with another filtering method, which proposed by Depovere's, in the term of MAE. The watermarks can be extracted after common image processing attacks with lower MAE value. Especially in case of enhancement operations with filtering and noise addition, increasing in performance is become more obvious.

\section{Acknowledgement}

This paper is extracted from research projects for Islamic Azad University, Firoozkooh branch, Firoozkooh, Iran.

\section{References}

1. Amirgholipour KS, Naghsh-Nilchi AR. A New Robust Digital Image Watermarking Technique Based on Joint DWT-DCT Transformation. ICCIT (Third International Conference on Convergence and Hybrid Information Technology). 2008; 2:539-44.

2. Amirgholipour KS, Naghsh-Nilchi AR. Robust Digital Image Watermarking Based on Joint DWT-DCT. International Journal of Digital Content Technology and its Applications. 2009; 3(2):42-54.

3. Amirgholipour KS, Mahfouzi M, Asfia M. A New Pre-processing Approach to Improve DCT-Based Watermarkings Extraction. International Association of Computer Science and Information Technology-Spring Conference, 2009. IACSITSC’09; 2009. IEEE, p. 131-5.

4. Amirgholipour KS, Sharifi AM. A Pre-Filtering Method to Improve Watermark Detection Rate in DCT based Watermarking. IAJIT. 2014 Mar; 11(2).

5. Potdar V, Han S, Chang E. A Survey of Digital Image Watermarking Techniques; Proc. of the IEEE International Conference on Industrial Informatics; 2005; Perth, Australia. p. 709-16.

6. Depovere G, Kalker T, Linnartz J-P. Improved watermark detection reliability using filtering before correlation. Proc. 5th IEEE Int. Conf. Image Processing, ICIP 98. 1998; 1:430-4.

7. Chu W. DCT-Based Image Watermarking Using Subsampling. IEEE Trans. Multimedia. 2003; 5(1):34-8.

8. Deng F, Wang B. A novel technique for robust image watermarking in the DCT domain. Proc. of the IEEE 2003 Int. Conf. on Neural Networks and Signal Processing. 2003; 2:1525-8.

9. Lin S, Chin C. A Robust DCT-based Watermarking for Copyright Protection. IEEE Trans. Consumer Electronics. 2000; 46(3):415-21.

10. Hsieh M, Tseng D, Huang Y. Hiding Digital Watermarks Using Multi-resolution Wavelet Transform. IEEE Trans. on Industrial Electronics. 2001; 48(5):875-82.

11. Reddy A, Chatterji B. A New Wavelet Based Logowatermarking Scheme. Pattern Recognition Letters. 2005; 26(7):1019-27.

12. Tay P, Havlicek J. Image Watermarking Using Wavelets; Proc. of the IEEE Midwest Symposium on Circuits and Systems; 2002; Oklahoma, USA. p. 258-61.

13. Watson AB. Perceptual optimization of DCT color quantization matrices. IEEE Int. Conf. Image Processing. 1994 Nov; $1: 100-4$.

14. Chou C-H, Li. Y-C. A perceptually tuned sub-band image coder based on the measure of just-noticeable distortion profile. IEEE Trans. Circuits and Systems for Video Technology. 1995 Dec; 5(6):467-76. 
15. Andrew WB, Yang GY, Solomon JA, Villasenor J. Visibility of wavelet quantization noise. IEEE Transactions on Image Processing. 1997; 6(8):1164-75.

16. Podilchuk CI, Zeng W. Image-adaptive watermarking using visual models. IEEE J. on Select. Areas Common. 1998 May; 16(4):525-39.

17. Lu, Chun-Shien, Shih-Kun H, Chwen-Jye S, Hong-Yuan ML. Cocktail watermarking for digital image protection. IEEE Transactions on Multimedia. 2000; 2(4): 209-24.

18. Barni, Mauro, Bartolini F, Piva A. Improved waveletbased watermarking through pixel-wise masking. IEEE Transactions on Image Processing. 2001; 10(5):783-91.

19. Kutter, Martin, Winkler S. A vision-based masking model for spread-spectrum image watermarking. IEEE Transactions on Image Processing. 2002; 11(1):16-25.

20. Fotopoulos V, Skodras AN. Improved watermark detection based on similarity diagrams. journal of Signal processing. Image communication. 2002; 17(4):337-45.

21. Braudaway GW, Mintzer F. Application of blurring filters to improve detection of invisible image watermarks. Security and watermarking of multimedia contents. Conference No5, Santa Clara CA , ETATS-UNIS. 2003 Jan; 5020:269-77.
22. Malik, Hafiz, Khokhar A, Ansari R. Improved watermark detection for spread-spectrum based watermarking using independent component analysis; Proceedings of the 5th ACM workshop on Digital rights management; 2005; ACM. p. 102-11.

23. Zhigeng P, Li L, Mingmin Z, David Z. Watermark Extraction by Magnifying Noise and Applying Global Minimum Decoder. Proceedings of the Third International Conference on Image and Graphics; 2004; IEEE.

24. Alirezanejad, Mehdi, Saffari V, Amirgholipour S, Sharifi AM. Effect of Locations of Using High Boost Filtering on the Watermark Recovery in Spatial Domain Watermarking. Indian Journal of Science and Technology. 2014; 7(4):517-24.

25. Amirgholipour S, Saffari V, Sharifi AM, Hasiri A. Effect of Different Places in Applying Laplacian Filter on the Recovery Algorithm in Spatial Domain Watermarking. Research Journal of Applied Sciences, Engineering and Technology. 2014; In Press.

26. Rafael GC, Woods RE. Digital Image Processing: Introduction. 2002. 